\title{
Influência do projeto estrutural $\epsilon$ da seleção dos materiais na pegada de carbono de uma estrutura de concreto armado
}

\author{
FERNANDA BELIZARI0-SILVA - Mestre, Pesquisadora \\ Unidade de NeGócios de HabitaCÃo e EdiFicacões, \\ Instituto de Pesquisas Tecnológicas do Estado de São Paulo - IPT \\ Escola Poltíćcica da USP
}

\begin{tabular}{|c|}
\hline DANIEL COSTA REIS - DouTOR, PESQUISADOR \\
\hline MARCO QUATTRONE - DoUTOR, PESQUISADOR \\
\hline VANDERLEY MOACYR JOHN - DOUTOR, PROFESSOR TITULAR \\
\hline $\begin{array}{l}\text { Departamento de Engenharia de Construç̄̃o CivlL, } \\
\text { Escola Politécnica da Universidade de São Paulo - Poli-USP }\end{array}$ \\
\hline
\end{tabular}

CARBONO DA ESTRUTURA, SEGUIDO PELA PEGADA DE CARBONO DO CIMENTO COMPOSTO. NO CENÁRIO QUE TAMBÉM CONSIDERA FORMULAÇÕES INOVADORAS, A ESCOLHA DA FORMULAÇÃO DO CONCRETO É A DECISÃO MAIS IMPORTANTE. EMBORA INOVAÇÕES POSSIBILITEM UMA MAIOR REDUÇÃO DAS EMISSÕES DE CARBONO, AS DECISÕES DO DIA A DIA DA CONSTRUÇÃO CAUSAM GRANDES VARIAÇÕES NA PEGADA DE CARBONO DAS ESTRUTURAS E, PORTANTO, DAS EDIFICAÇÕES, EVIDENCIANDO A IMPORTÂNCIA DOS AGENTES DA CONSTRUÇÃO PARA O DESENVOLVIMENTO SUSTENTÁVEL.
Reduzir a PEGADA dE CARBONO DE ESTRUTURAS DE CONCRETO ARMADO É ESSENCIAL PARA MITIGAR AS MUDANÇAS CLIMÁTICAS. ESTE TRABALHO QUANTIFICA A CONTRIBUIÇÃO DE DIFERENTES DECISÕES PARA A VARIAÇÃO DA PEGADA DE CARBONO DE UMA ESTRUTURA DE CONCRETO ARMADO. CONSIDERARAM-SE DECISÕES REFERENTES AO PROJETO ESTRUTURAL, À PRODUÇÃO DOS MATERIAIS E À CONSTRUÇÃO, PARA DOIS CENÁRIOS DE FORMULAÇÃO DO CONCRETO. NO CENÁRIO QUE CONSIDERA APENAS FORMULAÇÕES CONVENCIONAIS, O PROJETO ESTRUTURAL É O FATOR QUE MAIS CONTRIBUI PARA A VARIAÇÃO DA PEGADA DE o, projeto, inovação.

\section{INTRODUÇÃOO}

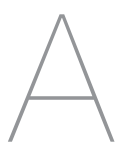

s mudanças climáticas representam o maior e mais urgente desafio a ser enfrentado pela humanidade. As atividades humanas já aumentaram a temperatura média do planeta em $1,1^{\circ} \mathrm{C}$ acima dos níveis pré-industriais e as consequências desse aumento já são sentidas por todos: ondas de calor extremo com secas prolongadas levam a uma epidemia de incêndios, chuvas intensas trazem enchentes catastróficas destruindo cidades medievais, ventos fortes em época de seca criam tempestades de poeira, etc. Não haverá local seguro se o planeta aquecer $3^{\circ} \mathrm{C}$. Esses efeitos negativos do aquecimento global custam vidas e perdas econômicas significativas. Apenas preparar a infraestrutura para resistir às mudanças climáticas não é suficiente: precisamos reduzir drasticamente as emissões de gases de efeito estufa ao longo dos próximos anos, chegando a emissões líquidas iguais a zero até 2050 (IPCC, 2021).

A indústria da construção representa mais de 10\% das emissões globais de $\mathrm{CO}_{2}$, sobretudo devido à produção de cimento e aço, am- bos matérias-primas de estruturas de concreto armado (UNEP, 2020). Sendo assim, as decisões referentes ao projeto e à produção dessas estruturas têm um impacto direto sobre o aquecimento global. O efeito do projeto fica evidente, por exemplo, no benchmark de pegada de carbono de estruturas desenvolvido por (DE WOLF et al., 2020) : para uma mesma tipologia de edifícios, a pegada de carbono ${ }^{1}$ pode variar em centenas de quilos de $\mathrm{CO}_{2 \mathrm{e}}$ por $\mathrm{m}^{2}$. A pegada de carbono da produção do concreto pode variar mais de $100 \mathrm{~kg} \mathrm{CO} / \mathrm{Cm}^{3}$ 
para uma mesma classe de resistência, dependendo da formulação (SILVA et al., 2019); se forem consideradas formulações inovadoras, essa diferença pode ser ainda maior (JOHN et al., 2018). A pegada de carbono do aço varia mais de $100 \mathrm{~kg} \mathrm{CO} / \mathrm{t}$, dependendo do conteúdo de material reciclado e da rota de fabricação (BAWDEN; WILLIAMS; BABBITT, 2016).

Entretanto, os estudos de redução da pegada de carbono de estruturas de concreto armado normalmente analisam os efeitos de decisões específicas de forma isolada. Dessa forma, não é possível saber qual decisão pode causar a maior alteração na pegada de $\mathrm{CO}_{2}$ das estruturas: seria o projeto? Ou a produção das matérias-primas? Qual a influência dos diferentes agentes do setor da construção na pegada de carbono das estruturas?

Sendo assim, o presente trabalho apresenta uma análise conjunta da influência de diferentes decisões de projeto, produção dos materiais e construção - na variação da pegada de carbono de uma estrutura de concreto armado. Este artigo é uma síntese do artigo "Stakeholder influence on global warming potential of reinforced concrete structure", publicado no Journal of Building Engineering (BELIZARIO-SILVA et al., 2021).

\section{MÉTODO}

Para avaliar a influência das diferentes decisões na variação da pegada de carbono de uma estrutura de concreto armado, realizaram-se as seguintes atividades:

a) Listaram-se as variáveis passíveis de serem influenciadas pelas decisões relativas ao projeto estrutural, fornecimento de materiais

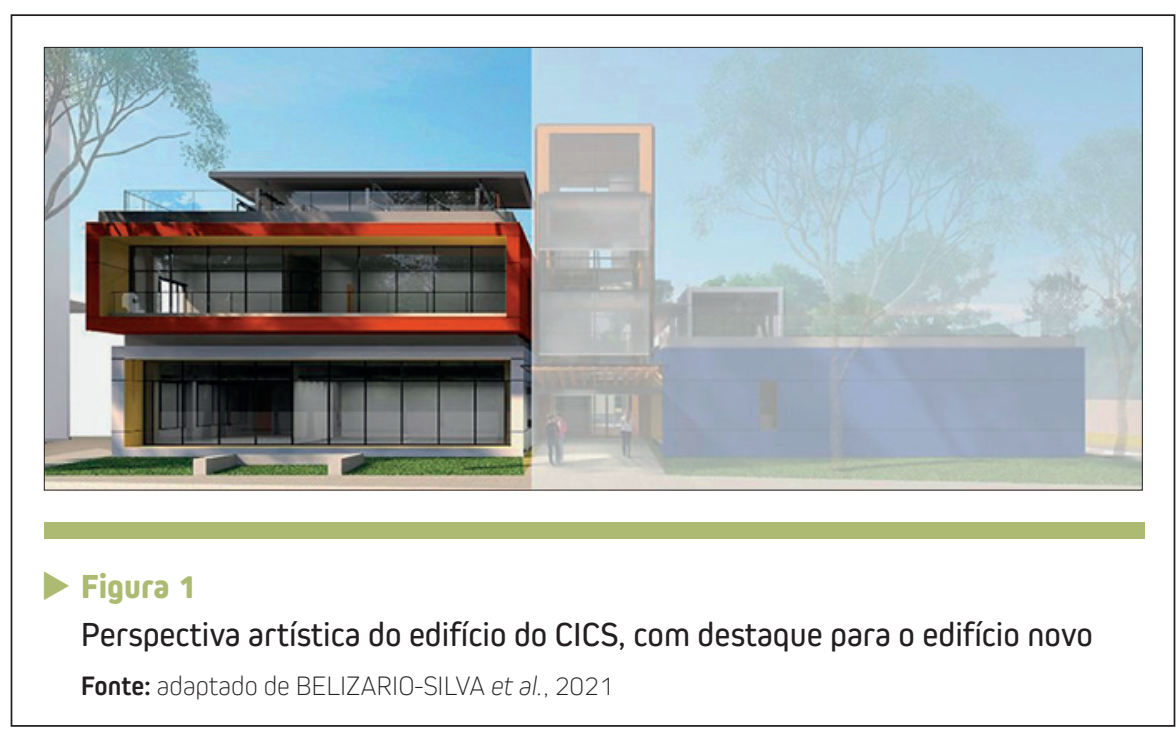

e construção, e quantificou-se a variação possível de cada uma dessas variáveis;

b) Calculou-se a pegada de carbono da estrutura e sua respectiva variação, por meio da propagação de incertezas pelo método de expansão de caos polinomial;

c) Calculou-se a contribuição de cada variável para a variação total da pegada de carbono da estrutura.

Essa análise foi feita para dois cenários referentes à formulação do concreto:

a) Concreto convencional: considera apenas as formulações convencionais;

b) Concreto convencional ou inovador: além das formulações de concreto convencionais, considera a opção de concreto com alto teor de filer, em substituição ao ligante, e baixo teor de água (High Filler Low Water - HFLM), o qual se encontra atualmente em desenvolvimento pela Escola Politécnica da USP.

A seguir, são descritos os principais procedimentos metodológicos adotados neste artigo. Os detalhes do método podem ser encontrados no artigo original.

\section{I Descrição do edifício}

O Centro de Inovação em Construção Sustentável (CICS) foi utilizado como estudo de caso (Figura 1). O CICS é um Living Lab da Escola Politécnica da USP, atualmente em construção, composto de três partes:

1) Um edifício novo;

2) Uma caixa de escada que conecta o edifício novo ao existente;

3) Um edifício existente que será reformado.

A estrutura analisada é a do edifício novo, que tem dois pavimentos e uma área total de $553 \mathrm{~m}^{2}$. Trata-se de uma estrutura pré-fabricada em concreto armado e protendido. Também foram consideradas as fundações, em estacas hélice contínua, que contêm um sistema de geotermia para climatização do edif́cio. No CICS, está sendo testado o concreto HFLW.

\subsection{Decisões e respectivas variáveis}

A seguir, são descritas as categorias de decisão analisadas nesse 
estudo e as respectivas variáveis que interferem no cálculo da pegada de carbono da estrutura.

\subsection{Projeto}

São consideradas três opções de laje pré-fabricada (Figura 2):

a) Laje alveolar de $16 \mathrm{~cm}$ de espessura, com vigas intermediárias;

b) Laje alveolar de $40 \mathrm{~cm}$ de espessura, sem vigas intermediárias;

c) Laje "pi" com $50 \mathrm{~cm}$ de espessura, sem vigas intermediárias.

Todas as lajes têm uma capa de concreto moldada in loco de $5 \mathrm{~cm}$ de espessura. As vigas de borda e colunas pré-fabricadas são iguais para as três opções de laje, assim como as estacas. Apesar disso, esses elementos foram mantidos no cálculo, pois interferem com as outras variáveis investigadas nesse estudo.

As opções de projeto estrutural interferem nas variáveis de quantidade de concreto e aço da estrutura, conforme mostra a Tabela 1. Para a caracteriza-

\section{Tabela 1 - Quantidades de material para cada opção de projeto de laje}

\begin{tabular}{|cccc}
\hline Material & $\begin{array}{c}\text { Laje alveolar } \\
16 \mathrm{~cm}\end{array}$ & $\begin{array}{c}\text { Laje alveolar } \\
40 \mathrm{~cm}\end{array}$ & $\begin{array}{c}\text { Laje pi } \\
50 \mathrm{~cm}\end{array}$ \\
\hline Concreto $25 \mathrm{MPa}\left(\mathrm{m}^{3}\right)$ - blocos & 28 & 28 & 28 \\
\hline Concreto $30 \mathrm{MPa}\left(\mathrm{m}^{3}\right)$ - estacas & 127 & 127 & 127 \\
\hline Concreto $30 \mathrm{MPa}\left(\mathrm{m}^{3}\right)$ - capa da laje & 44 & 44 & 44 \\
\hline Concreto $40 \mathrm{MPa}\left(\mathrm{m}^{3}\right)$ - pré-fabricado & 53 & 92 & 190 \\
\hline Concreto $50 \mathrm{MPa}\left(\mathrm{m}^{3}\right)$ - pré-fabricado & 61 & 141 & - \\
\hline Concreto $60 \mathrm{MPa}\left(\mathrm{m}^{3}\right)$ - pré-fabricado & 121 & - & - \\
\hline Concreto - total (m³) & 434 & 432 & 389 \\
\hline Aço - vergalhão $(\mathrm{t})$ - estacas e blocos & 9,1 & 9,1 & 9,1 \\
\hline Aço - vergalhão $(\mathrm{t})$ - estrutura & 27 & 19 & 28 \\
Aço - cabo de protensão $(\mathrm{t})$ & 4,8 & 5,3 & 2,6 \\
\hline Aço - total $\left(\mathrm{m}^{3}\right)$ & 40,9 & 33,4 & 39,7 \\
\hline
\end{tabular}

ção e propagação de incertezas, considerou-se que cada opção tem igual probabilidade de ocorrência (1/3).

\section{2.ट.2 MATERIAIS}

As variáveis consideradas para os materiais são apresentadas na Tabela 2. Elas contemplam decisões referentes à formulação do concreto, como, por exemplo, o teor de água, usado para calcular o teor de cimento, o qual irá interferir na pegada de carbono do concreto. Além disso, são consideradas as variações na pegada de carbono na produção dos materiais. Detalhes sobre as considerações feitas para se chegar aos intervalos de valores aqui apresentados
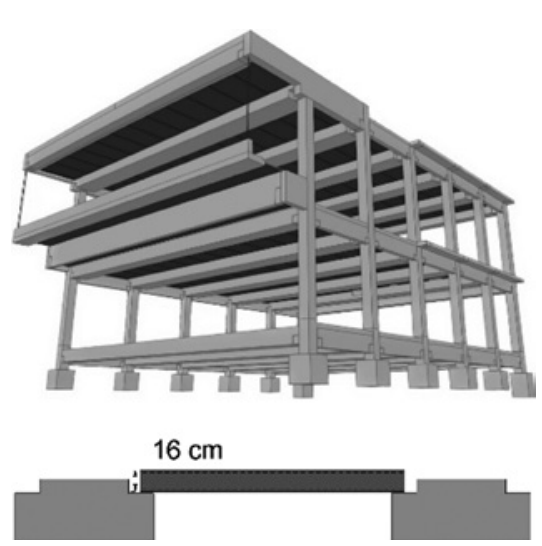

(2)
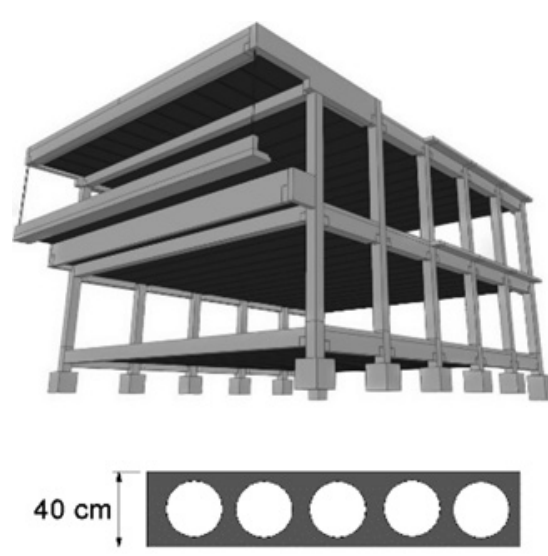

(b)
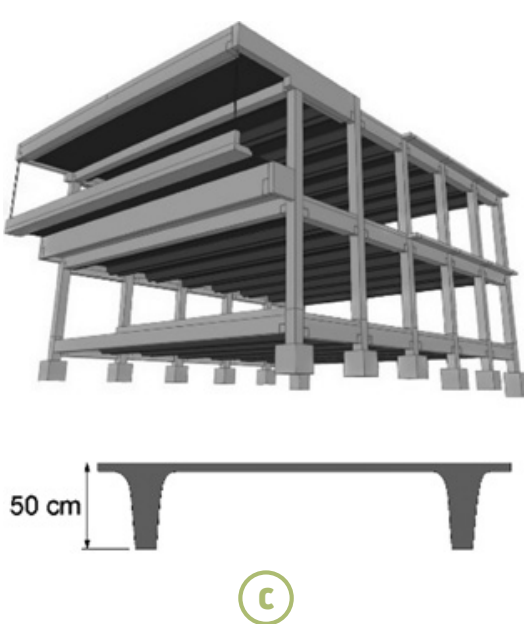

Figura 2

Representação 3D da estrutura, considerando as três opções de laje: (a) laje alveolar de 16 cm; (b) laje alveolar de $40 \mathrm{~cm}$; (c) laje "pi" de $50 \mathrm{~cm}$

Fonte: adaptado de BELIZARIO-SILVA et al., 2021 
estão disponíveis no artigo original.

\subsubsection{Construç̃ão}

A Tabela 3 apresenta as variáveis referentes à produção dos elementos pré-fabricados e à construção da estrutura.

\subsection{Estimativa da pegada de carbono da estrutura}

Todas as variáveis apresentadas no item 2.2 são dados de entrada para calcular a pegada de carbono da estrutura, do berço ao portão, ou seja, desde a extração dos recursos naturais até a estrutura pronta. Foram adotados alguns dados complementares da base de dados ecoinvent, como, por exemplo, a pegada de carbono do transporte rodoviário. O cálculo da pegada de carbono considerou o método indicado pelo Painel Intergovernamental de Mudanças Climáticas (IPCC), considerando um horizonte de 100 anos (GWP-100).

\subsection{Propagação de incertezas $\epsilon$ anális€ de sensibilidad $\epsilon$}

Todas as variáveis que interferem na pegada de carbono da estrutura foram modeladas como variáveis independentes. A incerteza de cada variável foi modelada por meio de sua distribuição de probabilidade. As incertezas foram propagadas por um método probabilístico denominado expansão de caos polinomial, por meio do software de análise de incertezas UQLab, o que permitiu calcular a variação total da pegada de carbono da estrutura, para cada um dos cenários de formulação do concreto.

Realizou-se, então, uma análise de sensibilidade para quantificar a contribuição de cada variável para a variação total da pegada de carbono da estrutura. Para isso, realizou-se uma análise de sensibilidade global, utilizando-se a técnica de decomposição de variância de Sobol. Detalhes dessa técnica
Tabela 2 - Variáveis referentes à produção e ao fornecimento dos materiais de construção

$\begin{array}{lll}\text { Variável } & \text { Valor* } & \begin{array}{c}\text { Distribuição de } \\ \text { probabilidade }\end{array}\end{array}$

Variáveis - concreto convencional

Dosagem do concreto

\begin{tabular}{|c|c|c|c|}
\hline \multicolumn{2}{|c|}{ Teor de água do concreto (L/m³) } & $180-200$ & Uniforme \\
\hline \multicolumn{2}{|c|}{ Resistência do cimento composto } & $\begin{array}{l}32 \mathrm{MPa}: 50 \% \\
40 \mathrm{MPa}: 50 \%\end{array}$ & 190 \\
\hline \multirow{5}{*}{ Teor de aditivo } & $\mathrm{f}_{\mathrm{ck}} 25 \mathrm{MPa}\left(\mathrm{kg} / \mathrm{m}^{3}\right)$ & $1,2-2,6$ & \multirow{5}{*}{ Uniforme } \\
\hline & $\mathrm{f}_{\mathrm{ck}} 30 \mathrm{MPa}\left(\mathrm{kg} / \mathrm{m}^{3}\right)$ & $1,4-3,0$ & \\
\hline & $\mathrm{f}_{\mathrm{ck}} 40 \mathrm{MPa}\left(\mathrm{kg} / \mathrm{m}^{3}\right)$ & $1,8-3,8$ & \\
\hline & $\mathrm{f}_{\mathrm{ck}} 50 \mathrm{MPa}\left(\mathrm{kg} / \mathrm{m}^{3}\right)$ & $2,3-4,6$ & \\
\hline & $\mathrm{f}_{\mathrm{ck}} 60 \mathrm{MPa}\left(\mathrm{kg} / \mathrm{m}^{3}\right)$ & $2,8-5,4$ & \\
\hline
\end{tabular}

Pegada de carbono do cimento CP-V-ARI $\left(\mathrm{kg} \mathrm{CO}_{2}\right.$ /t) Pegada de carbono do cimento composto $\left(\mathrm{kg} \mathrm{CO}_{2 \mathrm{e}} / \mathrm{t}\right)$ $770-840$ Uniforme Pegada de carbono dos agregados $\left(\mathrm{kg} \mathrm{CO}_{2 \mathrm{e}} / \mathrm{t}\right)$ $360-860$ Beta Pegada de carbono do vergalhão de aço $\left(\mathrm{kg} \mathrm{CO}_{2 e} / \mathrm{t}\right)$ $1,2-9,6$ Uniforme Pegada de carbono do cabo de protensão $\left(\mathrm{kg} \mathrm{CO}_{2 e} / \mathrm{t}\right)$ $980-2280$ Beta $\quad 1910-2570$

\section{Distância de transporte das matérias-primas}

Concreto dosado em central, fábrica-obra (km) Concreto pré-fabricado, fábrica-obra $(\mathrm{km})$ $4,5-11,4-28$ Uniforme

\begin{tabular}{|c|c|c|c|}
\hline \multicolumn{2}{|c|}{ Concreto pré-fabricado, fábrica-obra (km) } & $20-74-125$ & Triangular \\
\hline \multicolumn{2}{|c|}{ Vergalhão de aço, fábrica-obra (km) } & $56-521$ & Beta \\
\hline \multicolumn{2}{|c|}{ Cabo de protensão, fábrica-obra (km) } & $569-741$ & Uniforme \\
\hline \multicolumn{2}{|c|}{ Cimento, fábrica-fábrica (km) } & $300-500$ & Uniforme \\
\hline \multicolumn{2}{|c|}{ Agregados, fábrica-fábrica (km) } & $24-200$ & Uniforme \\
\hline \multicolumn{2}{|c|}{ Aditivos, fábrica-fábrica (km) } & $50-20000$ & Uniforme \\
\hline \multicolumn{4}{|c|}{ Variáveis adicionais - concreto inovador } \\
\hline \multicolumn{4}{|c|}{ Dosagem do concreto } \\
\hline \multirow{5}{*}{$\begin{array}{l}\text { Intensidade } \\
\text { de ligante }\end{array}$} & $\mathrm{f}_{\mathrm{ck}} 25 \mathrm{MPa}\left(\mathrm{kg} /\left(\mathrm{m}^{3} \cdot \mathrm{MPa}\right)\right)$ & $4,0-6,0-7,9$ & \multirow{5}{*}{ Triangular } \\
\hline & $\mathrm{f}_{\mathrm{ck}} 30 \mathrm{MPa}\left(\mathrm{kg} /\left(\mathrm{m}^{3} \cdot \mathrm{MPa}\right)\right)$ & $3,4-5,1-6,8$ & \\
\hline & $\mathrm{f}_{\mathrm{ck}} 40 \mathrm{MPa}\left(\mathrm{kg} /\left(\mathrm{m}^{3} \cdot \mathrm{MPa}\right)\right)$ & $2,7-4,1-5,4$ & \\
\hline & $\mathrm{f}_{\mathrm{ck}} 50 \mathrm{MPa}\left(\mathrm{kg} /\left(\mathrm{m}^{3} \cdot \mathrm{MPa}\right)\right)$ & $2,5-3,7-5,0$ & \\
\hline & $\mathrm{f}_{\mathrm{ck}} 60 \mathrm{MPa}\left(\mathrm{kg} /\left(\mathrm{m}^{3} \cdot \mathrm{MPa}\right)\right)$ & $2,5-3,7-5,0$ & \\
\hline
\end{tabular}

Fíler calcário, fábrica-fábrica $(\mathrm{km})$

100-1000

Uniforme 
Tabela 3 - Variáveis referentes à produção de pré-fabricados e à construção da estrutura

\begin{tabular}{|c|c|c|}
\hline Variável & Valor* & $\begin{array}{l}\text { Distribuição de } \\
\text { probabilidade }\end{array}$ \\
\hline $\begin{array}{l}\text { Pegada de carbono do consumo de energia no canteiro } \\
\qquad\left(\mathrm{kg} \mathrm{CO}_{2 \mathrm{e}} / \mathrm{m}^{2} \text { de área construída }\right)\end{array}$ & $0,1-25$ & Uniforme \\
\hline $\begin{array}{l}\text { Pegada de carbono do bombeamento de concreto in loco } \\
\qquad\left(\mathrm{kg} \mathrm{CO} \mathrm{e} / \mathrm{m}^{3} \text { concreto }\right)\end{array}$ & $0,3-2,7$ & Uniforme \\
\hline $\begin{array}{l}\text { Pegada de carbono da produção de pré-fabricados } \\
\qquad\left(\mathrm{kg} \mathrm{CO}_{2 \mathrm{e}} / \mathrm{m}^{3} \text { concreto) }\right.\end{array}$ & $5,2-112$ & Uniforme \\
\hline $\begin{array}{l}\text { Pegada de carbono das fôrmas para concreto moldado } \\
\qquad \text { in loco ( } \mathrm{kg} \mathrm{CO}_{2 \mathrm{e}} / \mathrm{m}^{3} \text { concreto) }\end{array}$ & $75-733$ & Uniforme \\
\hline Perdas de concreto - estacas hélice contínua (\%) & $3-20-35$ & Triangular \\
\hline Perdas de concreto - concreto moldado in loco (\%) & $2-10-25$ & Triangular \\
\hline
\end{tabular}

pegada de carbono da estrutura, para o concreto convencional e para o concreto inovador (HFLW).

\section{I Concreto convencional}

A pegada de carbono da estrutura considerando apenas o concreto convencional pode variar entre $162 \mathrm{e}$ 324 t $\mathrm{CO}_{2 e}$, ou seja, uma variação total de $100 \%$ (vide curvas contínuas da Figura 3). O fator que mais contribui para essa variação é a escolha do tipo de laje, que corresponde a 59\% da variância. Essa variável é importante porque determina as quantidades de concreto e aço da estrutura, parâmetros aos quais todas as demais variáveis estão associadas. Esse resultado demonstra a importância do projetista estrutural na definição da pegada de carbono dos edifícios.

A estrutura com laje "pi" tende a apresentar menor pegada de carbono (em média, $220 \mathrm{tCO}_{2 \mathrm{e}}$ ), sobretudo porque requer menor volume de concreto, seguida pelas lajes alveolares de $40 \mathrm{~cm}$ $\left(244 \mathrm{t} \mathrm{CO}_{2 \mathrm{e}}\right)$ e $16 \mathrm{~cm}\left(267 \mathrm{t} \mathrm{CO}_{2 \mathrm{e}}\right.$ ), respectivamente (vide curvas contínuas da Figura 3). Entretanto, as curvas de pro- babilidade para a pegada de carbono de cada opção de laje se sobrepõem, o que significa, por exemplo, que é possível ter uma estrutura com laje alveolar de $16 \mathrm{~cm}$ com desempenho ambiental comparável ao da estrutura com laje "pi", dependendo das demais decisões.

O segundo fator mais importante é a pegada de carbono do cimento composto, que corresponde a $23 \%$ da variância total. A variação da pegada de carbono do cimento composto (360 a $860 \mathrm{~kg} \mathrm{CO} / \mathrm{e} / \mathrm{t}$ ) se deve principalmente à variação do teor de clínquer desses cimentos (CP II, CP III e CP V). Ou seja, a escolha do tipo de cimento usado na formulação do concreto dosado em central é uma decisão importante para a pegada de carbono da estrutura; consequentemente, a escolha do fornecedor de concreto dosado em central em função de sua pegada de carbono também é importante.

O terceiro fator mais importante é o consumo de energia na produção dos elementos pré-fabricados de concreto, que corresponde a $8 \%$ da variância. Entretanto, essa variação está mais associada à incerteza dessa variável, estimada com base em dados secundários, do que a uma variação existente entre fabricantes. Os demais fatores não contribuem significativamente para a variação da pegada de carbono da estrutura. Ou seja, a diretriz bastante difundida de se utilizar materiais locais para reduzir impactos ambientais,

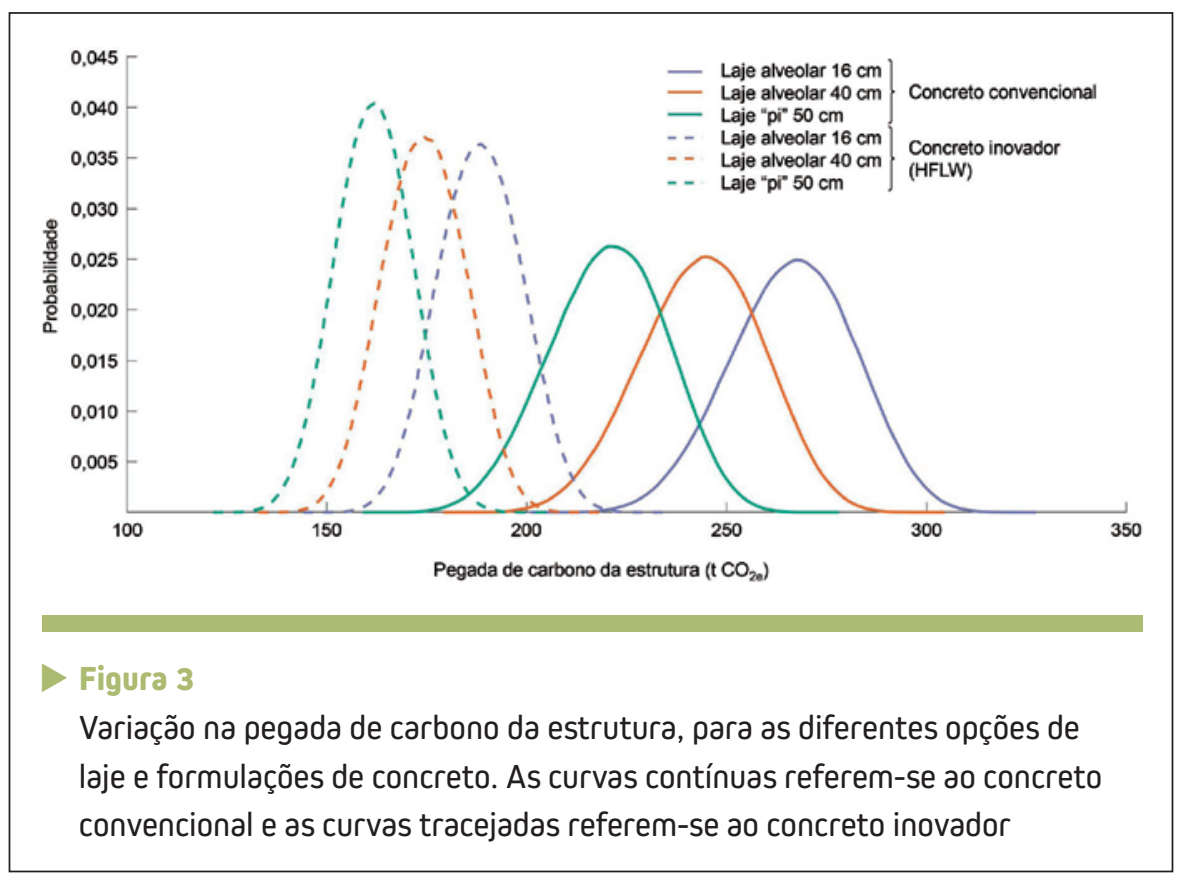




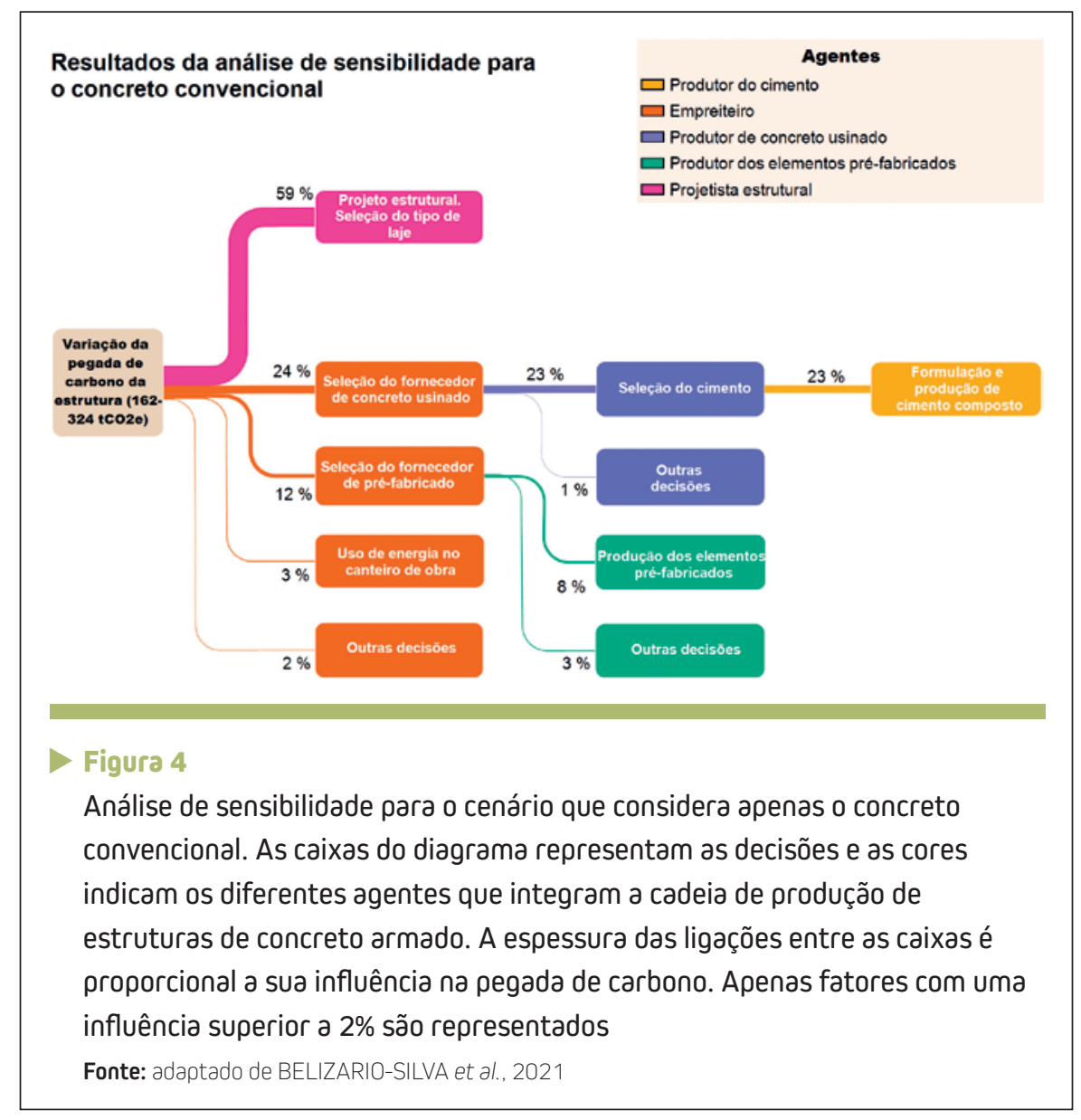

varia entre 124 e 324 t $\mathrm{CO}_{2 \mathrm{e}}$, ou seja, o limite mínimo é reduzido, pois o concreto HLFW tem uma menor pegada de carbono, como fica evidente nas curvas tracejadas apresentadas na Figura 3.

Nesse cenário, a variável mais importante é a escolha entre o concreto convencional e o concreto HFLW, que corresponde a 59\% da variância total (somando-se a escolha feita para o concreto dosado em central e para o concreto pré-fabricado). O projeto, por sua vez, é o segundo fator mais importante, representando $21 \%$ da variância. Observam-se dois grupos distintos de curvas de pegada de carbono, sendo que as menores pegadas correspondem ao concreto HFLW (curvas tracejadas) e as maiores, ao concreto convencional (curvas contínuas). Apesar disso, ainda é possível construir uma estrutura com concreto convencional

na prática tem pouco efeito sobre a pegada de carbono, visto que a variação na distância de transporte contribui com menos de 1\% da variância total.

\subsection{Concreto convencional ou inovador}

No cenário apenas com o concreto convencional, a decisão mais importante para a variação da pegada de carbono da estrutura é o projeto. As variáveis associadas à formulação do concreto contribuem muito pouco para essa variação. Entretanto, ao considerar que o concreto pode ser convencional ou inovador (HFLW), os resultados mudam significativamente. A pegada de carbono da estrutura

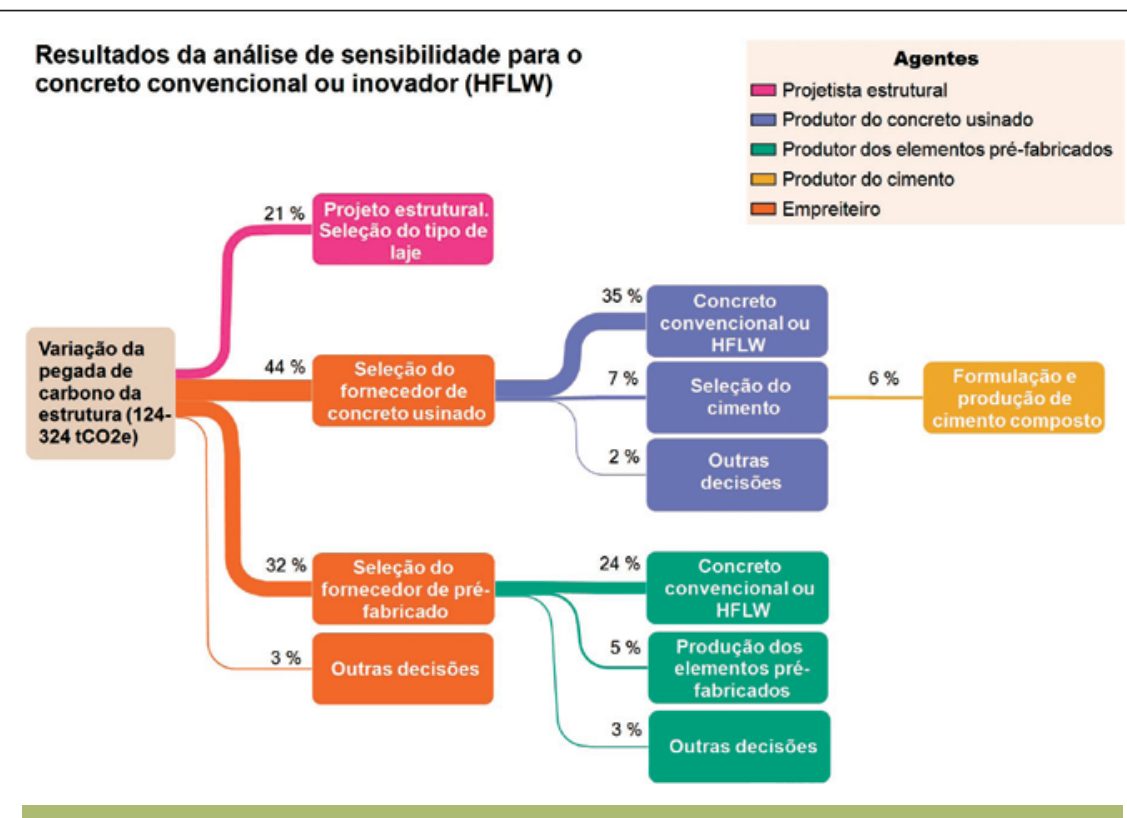

Figura 5

Análise de sensibilidade para o cenário que considera concreto convencional ou inovador. Apenas fatores com uma influência superior a $2 \%$ são representados Fonte: adaptado de BELIZARIO-SILVA et al., 2021 
e desempenho ambiental equivalente ao concreto HFLW, otimizando-se as demais decisões.

Portanto, a consideração de inovações na formulação do concreto permite reduzir ainda mais a pegada de carbono da estrutura e aumenta o protagonismo do produtor de concreto para melhorar o desempenho ambiental das estruturas. Observa-se que o artigo não explorou inovações em outras frentes, tais como abordagens inovadoras para o projeto (por exemplo, otimização topológica), para a produção de aço, entre outros. É de se esperar que tais inovações também aumentem o protagonismo de outros agentes do setor para ações de promoção do desenvolvimento sustentável.

\section{CONCLUSÕES}

Decisões que fazem parte do dia a dia de profissionais da construção podem alterar significativamente a pegada de carbono de estruturas de concreto armado e, consequen- temente, dos edifícios. O estudo de caso explorado nesse artigo mostra que a pegada de carbono pode dobrar (ou cair pela metade) em função dessas decisões. Se forem exploradas inovações, como o exemplo na formulação do concreto, essa variação pode ser ainda maior. O projeto estrutural e a escolha do cimento em função de sua pegada de carbono são as decisões mais importantes no cenário que considera apenas o concreto convencional, enquanto no cenário com concreto inovador, a escolha entre o concreto convencional e o concreto com alto teor de fíler calcário é a decisão que causa maior variação na pegada de carbono da estrutura.

Esses resultados demonstram a importância de que projetistas estruturais, fabricantes de materiais de construção e construtores considerem as implicações ambientais de suas decisões; do contrário, grandes oportunidades de mitigação das mudanças climáticas po- dem passar despercebidas. Para isso, é importante que indicadores de desempenho ambiental, como a pegada de carbono, passem a integrar os critérios de decisão do setor da construção.

\section{AGRADECIMENTOS}

Pesquisa contou com suporte da Fundação de Apoio ao Instituto de Pesquisas Tecnológicas INCT Tecnologias Cimentícias Eco-eficientes Avançadas (FAPESP $n^{\circ}$ 2014/50.948-3, CNPq n 485.340/ 2013-5). O projeto do Edifício experimental CICS é de Aflalo Gasperini sendo que estruturas e fundações cantam com o apoio de ArcelorMittal, InterCement, Leonardi Construções e Tupper. Marcelo Cuadrado, da Leonardi Construções Industrializadas, desenvolveu os projetos estruturais. Beatriz Gomes (Poli-USP) e Alina Galimshina, Maliki Moustapha e Guillaume Habert (ETH) participaram do desenvolvimento da versão original deste artigo.

\section{DREFERÊNCIAS BIBLIOGRÁFICAS}

[1] BAWDEN, K. R.; WILLIAMS, E. D.; BABBITT, C. W. Mapping product knowledge to life cycle inventory bounds: a case study of steel manufacturing. Journal of Cleaner Production, vol. 113, p. 557-564, Feb. 2016. D0l 10.1016/j.jclepro.2015.10.014. Available at: http://dx.doi.org/10.1016/j. jclepro.2015.10.014.

[2] BELIZARIO-SILVA, F.; GALIMSHINA, A.; REIS, D. C.; QUATTRONE, M.; GOMES, B.; MARIN, M. C.; MOUSTAPHA, M.; JOHN, V.; HABERT, G. Stakeholder influence on global warming potential of reinforced concrete structure. Journal of Building Engineering, vol. 44, no. July, p. 102979, Dec. 2021. DOI 10.1016/j.jobe.2021.102979. Available at: https://doi.org/10.1016/j.jobe.2021.102979.

[3] DE WOLF, C.; HOXHA, E.; HOLLBERG, A.; FIVET, C.; OCHSENDORF, J. Database of Embodied Quantity Outputs: Lowering Material Impacts Through Engineering. Journal of Architectural Engineering, vol. 26, no. 3, p. 04020016, Sep. 2020. D0I 10.1061/(ASCE)AE.1943-5568.0000408. Available at: http://ascelibrary.org/doi/10.1061/\%28ASCE\%29AE.1943-5568.0000408.

[4] IPCC. Summary for Policymakers. Climate Change 2021: The Physical Science Basis. [S. I.]: Cambridge University Press, 2021. Available at: https://www.ipcc.ch/report/ar6/wg1/.

[5] JOHN, V. M.; DAMINELI, B. L.; QUATTRONE, M.; PILEGGI, R. G. Fillers in cementitious materials - Experience, recent advances and future potential Cement and Concrete Research, vol. 114, p. 65-78, Dec. 2018. D0I 10.1016/j.cemconres.2017.09.013. Available at: https://linkinghub.elsevier com/retrieve/pii/S0008884616311425.

[6] SILVA, F. B.; OLIVEIRA, L. A.; YOSHIDA, O. S.; JOHN, V. M. Variability of environmental impact of ready-mix concrete: a case study for Brazil. IOP Conference Series: Earth and Environmental Science, vol. 323, p. 012132, 6 Sep. 2019. D0I 10.1088/1755-1315/323/1/012132. Available at: https://iopscience.iop.org/article/10.1088/1755-1315/323/1/012132.

[7] UNEP. 2020 Global Status Report for Buildings and Construction: Towards a Zero-emission, Efficient and Resilient Buildings and construction Sector Nairobi: [s. n.], 2020. 